\title{
Dense Capillary Discharge Plasma Waveguide Containing Ag Ions
}

\author{
Y. Wang, B. M. Luther, F. Pedaci, M. Berrill, F. Brizuela, M. Marconi, M. A. Larotonda, V. N. Shlyaptsev, and \\ J. J. Rocca, Fellow, IEEE
}

\begin{abstract}
Interferometry of plasmas generated by fast discharge excitation of $\mathrm{Ag}_{2} \mathrm{~S}$ microcapillary channels shows the formation of dense plasma waveguides capable of guiding intense laser beams. Discharge ablation of capillaries, $330 \mu \mathrm{m}$ or 440 $\mu \mathrm{m}$ in diameter, with $3-5.5-\mathrm{kA}$ current pulses formed concave plasma density profiles with axial electron density $>1 \times 10^{19}$ $\mathrm{cm}^{-3}$. These dense plasma waveguides containing highly ionized metal atoms are of interest to the development of a longitudinally pumped soft $X$-ray lasers.
\end{abstract}

$\mathbf{P}$ LASMA waveguides can extend the interaction length between intense laser pulses and plasmas beyond the limits imposed by diffraction and ionization induced refraction [1]. Several schemes for the generation of plasma waveguides based on either laser or discharge excitation have been studied. Some of these guides have been utilized in the development of soft $\mathrm{X}$-ray lasers [2]-[5]. However, plasma guides containing metal vapor ions that are of interest for the generation of laser amplification by collisional electron excitation at wavelengths below 20 $\mathrm{nm}$ remain to be developed. Herein, we report the use of a fast ( $\sim 55 \mathrm{~ns}$ first half-cycle) micro-capillary discharge (peak current 3-5.5 kA) for the generation of dense plasma waveguides containing a large concentration of Ag ions. The results are particularly relevant to the development of a longitudinally excited collisional soft X-ray laser at $13.9 \mathrm{~nm}$ in Ni-like Ag ions.

The plasmas were generated by discharge ablation of the walls of $\mathrm{Ag}_{2} \mathrm{~S}$ capillaries $330 \mu \mathrm{m}$ or $440 \mu \mathrm{m}$ in diameter and 2-4 mm in length. The discharge current pulses had a peak amplitude of 3-5.5 kA and a half-period of $\sim 55 \mathrm{~ns}$. To enhance the uniformity of the plasma column and its shot-to-shot reproducibility, a preplasma was generated by filling the capillary channels with 0.6 torr of $\mathrm{He}$ and preionizing the gas with a low current pulse. The plasma density distribution was measured using the third harmonic $(267 \mathrm{~nm})$ from a subpicosecond Ti:Sapphire laser system $(\sim 1 \mathrm{~mJ}, 800 \mathrm{~nm})$ in a Mach-Zender interferometer.

Fig. 1 shows a sequence of interferograms that map the temporal evolution of the electron density distribution within the

\footnotetext{
Manuscript received July 2, 2004; revised November 16, 2004. This work was supported in part by the U.S. Department of Energy Chemical Sciences, Geosciences, and Biosciences Division of the Office of Basic Energy Sciences, in part by the National Science Foundation under Grant ECS-9977677, in part by the National Science Foundation Engineering Research Center for Extreme Ultraviolet Science and Technology, and in part by the W. M. Keck Foundation.

Y. Wang, B. M. Luther, F. Pedaci, M. Berrill, F. Brizuela, M. Marconi, M. A. Larotonda, and J. J. Rocca are with the Electrical and Computer Engineering Department, Colorado State University, Fort Collins, CO 80523 USA (e-mail: bml@lamar.colostate.edu).

V. N. Shlyaptsev is with the Department of Applied Science, University of California-Davis, Livermore, CA 94551 USA.
}

Digital Object Identifier 10.1109/TPS.2005.845278
$400 \mu \mathrm{m}$ diameter $\mathrm{Ag}_{2} \mathrm{~S}$ capillary. It is observed that at these discharge conditions the time span during which concave electron density profiles are generated ranges from about $50-70 \mathrm{~ns}$ from the beginning of the current pulse. For times longer than $\sim 70$ ns, the concave density profile starts to degrade rapidly. It is of interest to notice that late in time, after a few half-periods of the current (e.g., $220 \mathrm{~ns}$ frame in Fig. 1), the curvature of the fringes reverses direction, indicating the presence of a convex electron density profile with maximum density on axis.

Fig. 2(a) shows an on-axis interferogram corresponding to a $440 \mu \mathrm{m}$ diameter $2.2 \mathrm{~mm}$ long $\mathrm{Ag}_{2} \mathrm{~S}$ capillary excited by a 5.2 $\mathrm{kA}$ current pulse, obtained $59 \mathrm{~ns}$ after the initiation of the current pulse. Fig. 2(b) shows an overlay of the experimental interferograms and interferograms computed from an electron density profile that was adjusted to fit the experimental data. The resulting radial electron density profile found by this procedure is shown in Fig. 2(c). The electron density difference between the axis of the channel and the vicinity of the walls is measured to be $\Delta \mathrm{Ne} \sim 1.0 \times 10^{19} \mathrm{~cm}^{-3}$. The absolute value of the electron density on axis was determined measuring the absorption of a 267 -nm wavelength probe beam of $\sim 100-\mu$ m diameter propagated along the capillary axis assuming an electron temperature of $20 \mathrm{eV}$ and a mean ionization of $\mathrm{Z}=8.6$ estimated from the code RADEX. The measured absorptions for the majority of the strongly guided shots $(\sim 50-70 \mathrm{~ns})$ amount to axial electron densities of $\sim 2-3 \times 10^{19} \mathrm{~cm}^{-3}$. The discharges through $330-\mu \mathrm{m}$ diameter capillaries were measured to generate plasmas with higher electron density on axis and steeper density walls. The measured electron density profile for a 440- $\mu \mathrm{m}$ diameter capillary shown in Fig. 2 can be computed to guide a beam with a matched mode size of $\omega_{0}=27 \mu \mathrm{m}$. Beam propagation experiments were conducted focusing 120 ps, 100 mJ, Ti: Sapphire laser pulses at the entrance of the capillary channel into a $\omega_{0} \sim$ $30-\mu \mathrm{m}$ spot. Exit mode patterns corresponding to a $440-\mu \mathrm{m}$ diameter, 4-mm-long capillary, showed an output mode pattern with a well defined peak of $\sim 54 \mu \mathrm{m}$ full-width half-maximum (FWHM) and a relatively low pedestal.

\section{REFERENCES}

[1] C. G. Durfee, III et al., "Development of a plasma waveguide for high intensity laser pulses," Phys. Rev. E, Stat. Phys. Plasmas Fluids Relat. Interdiscip. Top., vol. 51, pp. 2368-2389, 1995.

[2] K. A. Janulewicz et al., "Demonstration of a hybrid collisional soft-X-ray laser," Phys. Rev. A, Gen. Phys., vol. 63, p. 033 803, 2001.

[3] D. V. Korobkin et al., "Demonstration of soft X-ray lasing to ground state in Li III," Phys. Rev. Lett., vol. 77, p. 5206, 1996.

[4] A. Butler et al., "Demonstration of a collisionally excited optical-fieldionization XUV laser driven in a plasma waveguide," Phys. Rev. Lett., vol. 91, p. $205001,2003$.

[5] J. Hopwood, "Review of inductively coupled plasmas for plasma processing," Plasma Sources Sci. Technol., vol. 1, pp. 109-116, 1992. 

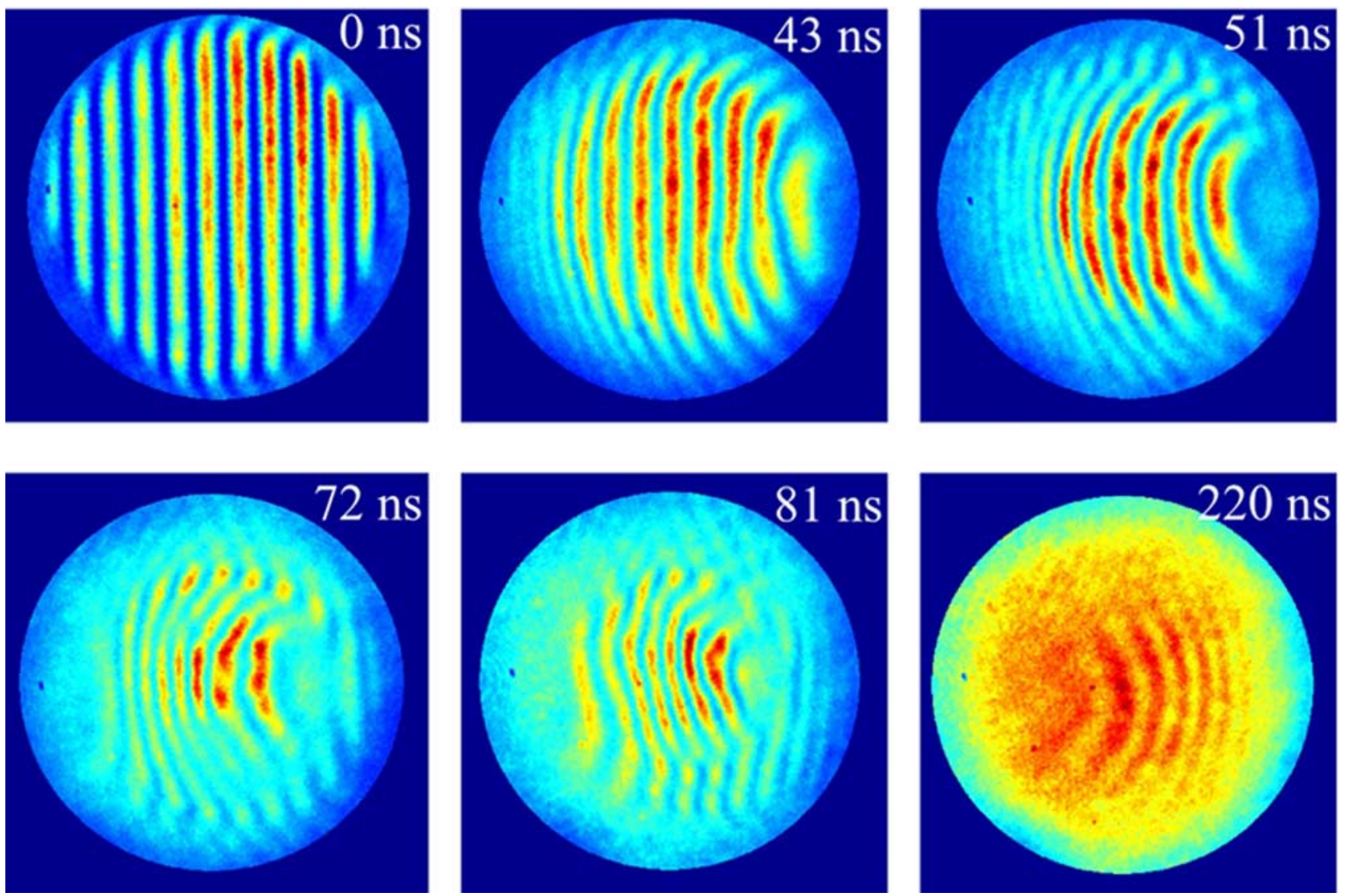

Fig. 1. Shown is a sequence of interferograms corresponding to a $440 \mu \mathrm{m}$ diameter $\mathrm{Ag}_{2} \mathrm{~S}$ capillary. Times are measured with respect to the beginning of the current pulse.

A

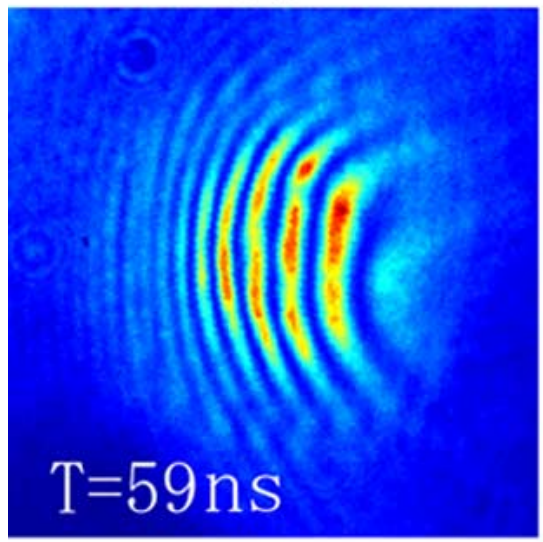

$\mathrm{B}$

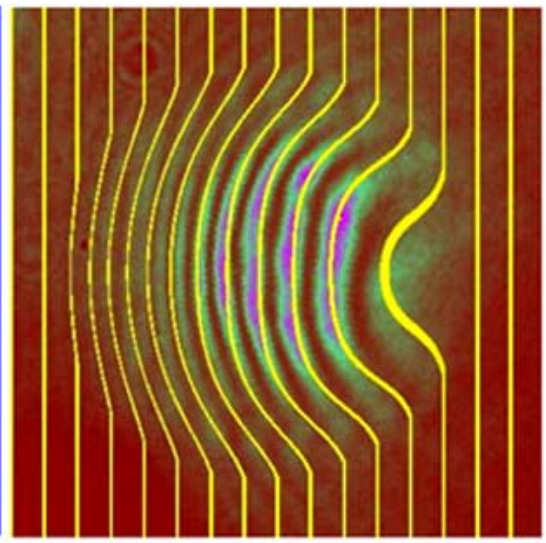

C

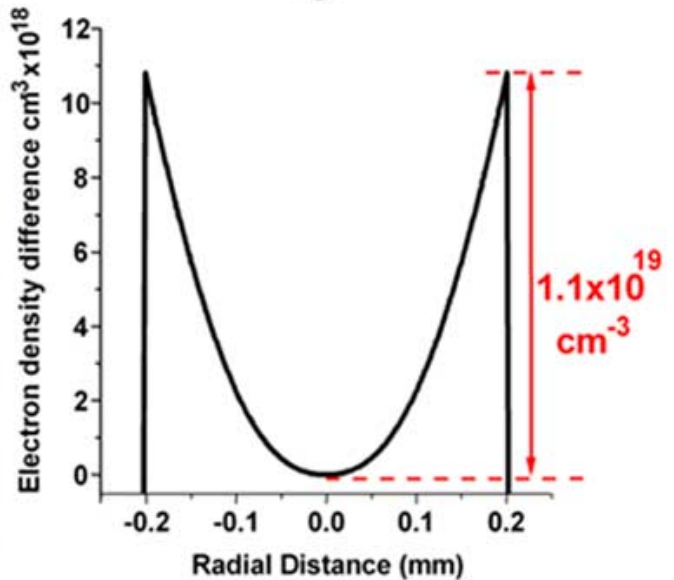

Fig. 2. (A) Interferograms of a 440- $\mu \mathrm{m}$-diameter, 2.2-mm-long, $\mathrm{Ag}_{2} \mathrm{~S}$ capillary discharge plasma $59 \mathrm{~ns}$ after the initiation of the current pulse. (B) Overlay of measured and best-fit simulated interferogram. (C) Corresponding radial variation of the electron density distribution. Note that the on-axis electron density value was determined from absorption measurements. 